\title{
Estado de los servicios de atención sobre salud mental en Tunja, 2017
}

\author{
Status of the mental health care services in Tunja, 2017
}

Estado de serviços de saúde mental na cidade de Tunja, 2017

\author{
Adriana Marcela Mesa-Fernández* \\ Yenny Salamanca-Camargo **
}

\section{Resumen}

Objetivo: Describir el estado actual de los servicios de atención en salud mental en la ciudad de Tunja. Materiales y método: Investigación de tipo descriptivo y corte trasversal, en la que a partir de dos fichas diseñadas por las autoras, conforme a las disposiciones planteadas por las resoluciones 2003 de 2014 y 6408 de 2015 del Ministerio de Salud y Protección Social, con las que se pretendió verificar el cumplimento en acceso, cobertura, procedimiento, condición de habilitación de servicios y prestación de atención de salud mental, en nueve Entidades Administradoras de Planes de Beneficios (EAPB) y 32 Instituciones prestadoras de servicios (IPS), vinculadas al directorio de salud de la Secretaria de Protección Social de Tunja. Resultados: Ocho EAPB cuentan con habilitación de servicios en salud mental, la mayoría tiene convenio con el Centro de rehabilitación integral de Boyacá (CRIB); ocho de las 32 IPS brindan el servicio de urgencias para salud mental; 22 el servicio de consulta externa y ninguna cuenta con el servicio de hospitalización en unidad especializada para consumidores de sustancias psicoactivas. Conclusiones: Los servicios habilitados para salud mental son limitados y no cumplen con la totalidad de disposiciones propuestas en las resoluciones; lo anterior se ve reflejado principalmente en la carencia de profesionales, en virtud que se requiere de profesionales especialistas, con competencias que les permitan brindar servicios oportunos y de calidad.

Palabras clave: Servicios de salud mental, habilitación sanitaria.

\section{Autor de correspondencia}

* $ه$ Psicóloga. Investigadora grupo de investigación clínica y salud, Universidad Pedagógica y Tecnológica de Colombia. Correo: adrianamarcela.mesa@uptc.edu.co. (D) https://orcid.org/0000-0001-8461-6942. Tunja, Colombia.

** Master Internacional en Psicología clínica y de la Salud. Magíster en psicología - énfasis clínico. Coordinadora grupo de investigación clínica y salud. Docente Universidad Pedagógica y Tecnológica de Colombia. Correo: yenny.salamanca@uptc.edu.co. (i) https://orcid.org/0000-0002-0928-8907. Tunja, Colombia.

\begin{abstract}
Objective. Describe the current status of mental health care services in the city of Tunja Materials and method. Descriptive and cross-sectional research, parting from two data sheets designed by the authors, according to the regulations established by the resolutions 2003 of 2014 and 6408 of 2015 from
\end{abstract}

Este es un artículo bajo la licencia CC BY (https://creativecommons.org/ licenses/by/4.0/) (c) (i) 
ISSN-PRINT

1794-9831

E-ISSN 2322-7028

Vol. 15 No. 2

Jul - Dic 2018

Cúcuta, Colombia

the Ministry of Health and Social Protection, with the intention of verifying the compliance of access, coverage, procedure, habilitation services and provision of care for mental health, in nine Administrative Entities of Beneficial Plans (EAPB) and 32 health facilities (IPS), linked to the health directory of the Secretary of Social Protection of Tunja. Results. Eight EAPB count on with mental health habilitation services, most of them with an agreement with the Comprehensive Rehabilitation Facility of Boyaca (CRIB); on the other hand, eight of the 32 IPS (health facilities) provide the emergency service for mental health; 22 the outpatient service and none has the service of hospitalization in a specialized unit for users of psychoactive substances. Conclusions. The enabled services for mental health are limited and do not meet with the totality of regulations proposed by the resolutions; the former is mainly reflected in the lack of professionals, in virtue of the requirement of specialists with capacities that allow them to provide opportune and quality services.

Keywords: Mental health services, sanitary habilitation.

\section{Resumo}

Objetivo: Descrever o estado atual dos serviços de saúde mental na cidade de Tunja, estado de Boyacá. Materiais e métodos. Pesquisa de tipo descritivo e corte transversal, na qual a partir de duas fichas projetadas pelas autoras, conforme às disposições levantadas pelas resoluções $2003 \mathrm{de}$ 2014 e 6408 de 2015 do Ministério de Saúde e Proteção Social, com as que se pretendeu verificar o cumprimento em aceso, cobertura, procedimento, condição de qualificação de serviços e prestação de atenção de saúde mental, em nove Entidades Administradoras de Planos de Benefícios (EAPB) e 32 Instituições prestadoras de serviços (IPS), ligadas ao diretório de saúde da Secretaria de Proteção Social de Tunja. Resultados: Oito EAPB contam com habilitação de serviços em saúde mental, a maioria tem convenio com o Centro de Reabilitação Integral de Boyacá (CRIB); por outro lado, oito das 32 IPS fornecem o serviço de urgências para saúde mental; 22 o serviço de consulta externa e nenhuma conta com o serviço de hospitalização em unidade especializada para consumidores de sustâncias psicoativas. Conclusões: Os serviços autorizados para saúde mental são limitados e não cumprem com a totalidade das disposições propostas nas resoluções; assim o anterior se vê refletido principalmente na carência de professionais, em virtude de que se requer de professionais especialistas, com competências que lhes permitam fornecer serviços oportunos e de qualidade.

Palavras-chave: Serviços de saúde mental, habilitação sanitária.

\section{Introducción}

Los trastornos mentales van en aumento, siendo un factor de riesgo marcado por la situación de pobreza. Las cifras presentadas en los últimos 12 meses muestran que: el $4.7 \%$ de la población infantil tiene algún trastorno mental, el $4.4 \%$ de los adolescentes tienen cualquier trastorno mental y $4 \%$ de los adultos han tenido algún trastorno mental; por consiguiente, las personas con bajos ingresos económicos se encuentran con un grado de vulnerabilidad mayor $(1,2)$.

Según la Organización Mundial de la Salud (OMS) (3), los trastornos mentales son enfermedades prevalentes en la sociedad, constituyen un problema de salud con gran impacto en la economía, la discapacidad, la dependencia y los aspectos sanitarios. Al respecto, Santacruz et al. (4) exponen que la salud mental es un fenómeno complejo determinado por la integración de diferentes variables individuales, sociales y ambientales, indicando que existen modelos integrales de atención orientados a la protección de este tipo de enfermedad.

Al respecto, Honorato et al. (5), señalaron que actualmente las enfermedades en salud mental están siendo abordadas mediante las intervenciones propuestas por el sistema sanitario, situación que ha generado una gran carga para los sistemas de salud disminuyendo su eficiencia, debido sobre todo a la ausencia de una estructuración eficiente y a una atención clínica fundamentada en la Atención Primaria enSalud(APS). Por supartela OMS (6,7) expone que hay escasez de recursos en los países para atender las necesidades de salud mental y de una inadecuada distribución, dándoles un uso ineficiente; así mismo, advierte que el número de profesionales sanitarios que se encargan de brindar servicios en salud mental, son pocos, especialmente en países con menos recursos, añadiendo que en algunas ocasiones no se encuentran capacitados 
para brindar servicios específicos, disminuyendo la calidad del servicio médico; en otros casos, la ejecución de los procedimientos son contraproducentes .

Con el fin de analizar cómo se desenvuelven los sistemas de salud más eficientes, Álvarez (8), realizó un análisis en 48 países desarrollados, teniendo en cuenta criterios tales como esperanza de vida, coste de salud y coste absoluto por capital del sistema de salud. El análisis determinó que los países asiáticos son los primeros en la lista, sobre todo Hong Kong, Singapur y Japón, al contar con un gobierno que controla el sistema de salud; además, debido a su excelente economía, la mayoría de los servicios en salud están financiados por el sistema público. El segundo puesto lo ocupa España, considerado como el estado más eficiente de Europa y el quinto lugar en el mundo, caracterizado por tener una sanidad pública financiada por los impuestos con cobertura universal.

González (9) por su parte, realizó un análisis comparativo de los sistemas de salud de Cuba, Canadá y Colombia, encontrando que para el caso de Canadá, el sistema sanitario responde a las necesidades particulares de salud de la población, existe igualdad de acceso a los servicios de salud, implementa varios programas de vigilancia, prevención y control de las enfermedades y ofrece un sistema donde es posible recibir atención médica aun estando en el extranjero; por su parte Cuba, tiene un sistema de salud universal, gratuito y la prestación de los servicios se encuentra enfocada en la estrategia de APS con enfoque integral, garantizando la accesibilidad de la población a los servicios; sin embargo, teniendo en cuenta que en Colombia no se cuenta con características que se asemejaran a las de los países anteriores, no fue posible comparar su sistema sanitario.

Si bien, Colombia aun presenta dificultades en su sistema sanitario, es importante aclarar que se han implementado estrategias como las legislaciones que abarcan salud y específicamente salud mental, teniendo como fin, la disminución de los riesgos en sus habitantes; dentro de estas, se encuentra la Ley 100 de 1993 (10) la cual garantiza el derecho a la protección integral de la salud en todos los niveles de atención, con especial énfasis en los aspectos de promoción, prevención y fomento de la salud, sin descuidar el diagnóstico oportuno, el tratamiento y la rehabilitación de las enfermedades en la cantidad, calidad y eficiencia requeridas.

Así mismo, está la ley 1616 de $2013(11,12)$ cuyo objetivo es garantizar el derecho a la salud mental, priorizando la de los niños(as) y adolescentes de la población colombiana, mediante la promoción de la salud y la prevención de enfermedades mentales. Por su parte, la ley 1751 de 2015 (13), regula el derecho fundamental a la salud y establece los mecanismos de protección necesarios para garantizar la integridad en la atención a través de la promoción, prevención, diagnóstico, tratamiento, recuperación, rehabilitación y disminución de la enfermedad; de otra parte, la ley 1566 de 2012 (14), expone las condiciones necesarias que garantizan la atención integral de personas que consumen sustancias psicoactivas.

Específicamente en el departamento de Boyacá, la Ordenanza 023 de 2010 (15), adopta el modelo de APS con el enfoque en salud familiar (SF); tiene como fin optimizar e integrar el Sistema General de Seguridad Social en Salud (SGSSS); igualmente, promueve una política pública en salud, mediante la articulación y armonización del aseguramiento, la prestación de servicios en salud y el desarrollo de políticas y programas en Salud Pública soportadas en procesos de gestión social y en una política de carácter intersectorial.

Al analizar estudios relacionados con la evaluación de los servicios sobre salud mental, que aunque son escasos, se encuentran dos que presentan semejanzas con la presente investigación. Tuvieron en cuenta la percepción de los usuarios y su conocimiento frente a los servicios de salud mental, demostrando que en su mayoría la perciben como resultado de hechos de diferente naturaleza, y al mismo tiempo la consideran de difícil acceso debido al tipo y a la cantidad de afiliaciones al SGSSS (16). Así mismo, exponen que hay insuficiencia de presupuesto y de personal calificado que atienda estos servicios; teniendo en cuenta estas limitaciones se propone formular políticas que enfrenten el problema de los trastornos mentales, mediante la información correcta que genere acciones para así tomar decisiones coherentes que permitan un avance en solucionar y mejorar los problemas relacionados con la salud mental y todo lo que tenga que ver con este tema; del mismo modo, se busca contar con el interés del sistema político para que solucione las problemáticas sobre este tópico presentes en la población (17).

Aunque en la actualidad se observa un avance hacia el acceso a los servicios en salud mental, es notorio que en un estudio realizado por el Ministerio de Salud y Protección Social en colaboración con Colciencias y la Pontificia Universidad Javeriana (1), se sugiere sobre la importancia de lograr el acceso real a los servicios en salud 
mental desde una perspectiva comunitaria, teniendo en cuenta la estrategia de APS, su promoción y prevención, enfatizando en la construcción y estructuración de una ruta integral de atención en salud mental en todo el país, que permita el acceso y atención eficiente en todos los aspectos relacionados con este tipo de enfermedad.

Si bien, Colombia cuenta con un soporte legislativo sobre salud mental, su implementación y cumplimiento es insuficiente, debido al escaso conocimiento que se tiene desde los entes competentes y la población.

\section{Objetivos}

\section{Objetivo general}

- Describir el estado actual de los servicios de atención en salud mental brindados por las Instituciones Prestadoras de Servicio (IPS) y Entidades Administradoras de Planes de Beneficios (EAPB) de Tunja, a partir del cumplimiento de las disposiciones propuestas en las resoluciones 2003 de 2014 y 5592 de 2015.

\section{Objetivos específicos}

- Identificar los servicios de salud mental habilitados por las EAPB en la ciudad de Tunja.

- Analizar el cumplimento de las condiciones de habilitación por parte de los servicios de salud mental brindados en la red de prestadores de servicios de salud de Tunja

\section{Materiales y Métodos}

Se realizó una investigación de tipo descriptivo y corte trasversal, para la cual se diseñaron dos fichas que evalúan los servicios habilitados $\mathrm{y}$ ofertados sobre salud mental en la ciudad de Tunja, para ser aplicadas tanto en las EAPB presentes en el directorio de servicios de salud de la Secretaria de Protección Social, como en las IPS vinculadas a la red de servicios contratada. La información se recolectó a partir de una entrevista realizada a profesionales designados por cada institución, mediante una carta dirigida a cada una de las entidades firmada por el Secretario de Protección Social de la ciudad de Tunja, en la que se exponía el objetivo de la investigación, también se solicitaba el consentimiento informado para acceder y recolectar la información correspondiente a las fichas diseñadas.

Posterior a ello se diseñó una base de datos con la información recolectada; igualmente, se realizó un análisis, mediante la interpretación de los datos recolectados a partir de estadísticos descriptivos como media, moda, frecuencia de las variables cuantitativas y análisis de las variables cualitativas a través de los programas de Microsoft Excel y el estadístico SPSS 22.0.

A continuación se describen los componentes de las fichas diseñadas para el estudio.

- Ficha de Evaluación IPS, de autoría propia, que contó con el apoyo y la revisión de la Secretaria de Protección Social de Tunja. Esta ficha fue diseñada a partir de la resolución 2003 de 2014 del Ministerio de Salud y Protección Social dividida en diez apartados diferentes: datos específicos sobre la IPS y la red de contratación para el servicio de psicología, el tipo de aseguramiento, servicios en salud mental, protocolos, consulta externa, acompañamiento en otros procedimientos, atención domiciliaria y ambulatoria, apoyo diagnóstico, hospitalización y hospitalización específica para consumidores de SPA. Contiene las instrucciones en cada apartado para su buen desarrollo y está conformada por 112 ítems, de los cuales 83 son de respuesta dicotómica y 29 de respuestas abiertas.

- Ficha de evaluación EAPB, de autoría propia, contó con el apoyo y la revisión del equipo de trabajo de salud mental de la Secretaria de Protección Social de Tunja; diseñada a partir de la resolución 5592 del 2015 y de su actualización, la resolución 6408 del 2016 del Ministerio de Salud y Protección Social. Se divide en cinco apartados entre los que se encuentran: datos específicos de la EAPB así como de la red contratada de IPS para atención en salud mental, tipo de aseguramiento y servicio de urgencias, perfil del profesional contratado en salud mental, otros servicios complementarios en salud mental y servicios prestados en una edad específica. Incluye instrucciones para cada apartado y consta de 50 ítems, de los cuales 28 son de respuesta dicotómica y 22 de respuesta abierta.

Teniendo en cuenta que esta investigación busca realizar un aporte para el desarrollo de la psicología y el bienestar humano, se garantiza el cumplimiento de la norma ética, teniendo en cuenta fundamentalmente la Ley 1090 del año 2006 y la resolución 8430 de 1993, donde se expone que los investigadores deben ser objetivos ante 
los hallazgos, usándolos de manera adecuada. Se envió una carta emitida por la Secretaria de Protección Social en donde se da a conocer a cada una de las instituciones entrevistadas el objetivo de la investigación y la manera de compilación, uso y manejo ético de la información, así como la generación de espacio para la respuesta a las inquietudes; también, se les permitió a las instituciones que fueran autónomas en su participación o no del estudio $(18,19)$.

\section{Resultados}

Teniendo en cuenta los objetivos propuestos en esta investigación se observa que respecto a las EAPB y de acuerdo al directorio de servicios de salud de la Secretaria de Protección social de Tunja, se encuentran registradas 12 , de las cuales, se pudo acceder a nueve para obtener la información. Respecto al tipo de aseguramiento, se observa que habilitan sus servicios de salud mental específicamente para el tipo de aseguramientos subsidiado y contributivo; en cuanto a lo relacionado con los servicios habilitados de psicología, ocho de las nueve entidades visitadas cuentan con este servicio, razón por la cual es importante aclarar que en adelante se analizarán solamente los resultados a partir de estas instituciones que tienen habilitados los servicios de salud mental.

Por otra parte, la red de IPS contratadas para prestación de servicio en psicología, la que posee más convenios con las EAPB de Tunja es el Centro de Rehabilitación Integral de Boyacá (CRIB) que cuenta con cinco, la SIREB con cuatro y otras de las entidades tienen convenios en Boyacá y Cundinamarca (Figura 1).

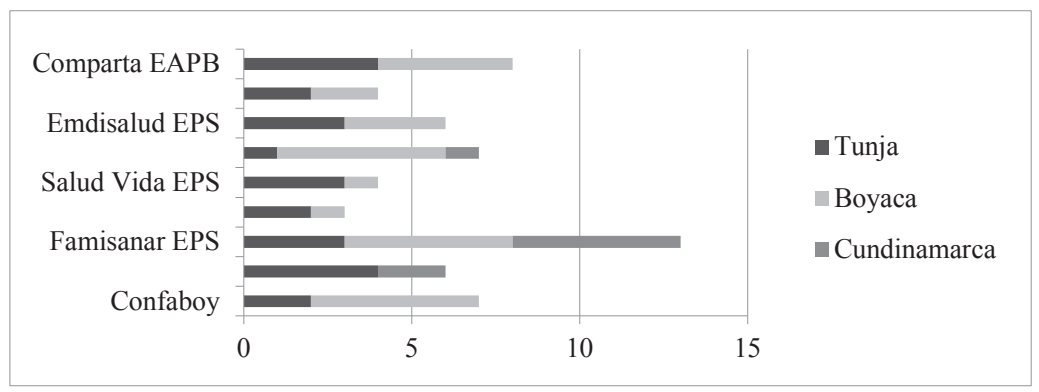

Figura 1. Número de Convenios que tiene cada EAPB para prestación de servicios de psicología

Fuente: Elaboración propia

En relación con la habilitación para servicios de urgencias en salud mental, ocho de las entidades cuentan con la respectiva habilitación y los servicios que ofrecen se relacionan con hospitalización, rehabilitación y atención psicológica. En cuanto a las instituciones que prestan el servicio de urgencias de salud mental, cuentan con entre uno y cinco convenios (Tabla 1).

Tabla 1. Convenio de las instituciones que prestan el servicio de urgencias para salud mental

\begin{tabular}{|c|c|c|c|c|c|c|}
\hline \multirow{2}{*}{ EAPB } & \multicolumn{6}{|c|}{ Remisión urgencias } \\
\hline & CRIB & Hospital San Rafael & Clínica Medilaser & Clínica Santa Teresa & Boyacá & Cundinamarca \\
\hline Comfaboy & $\mathrm{X}$ & & & & & \\
\hline Sanitas EPS & $\mathrm{X}$ & $\mathrm{X}$ & $\mathrm{X}$ & & & \\
\hline Famisanar EPS & & & & & & $\mathrm{X}$ \\
\hline Comfamiliar EPS & $\mathrm{X}$ & $\mathrm{X}$ & & & & \\
\hline Salud Vida EPS & & $\mathrm{X}$ & & $\mathrm{X}$ & & \\
\hline Coomeva EPS & & & $\mathrm{X}$ & & $\mathrm{X}$ & \\
\hline \multicolumn{7}{|l|}{ Emdisalud EPS } \\
\hline Nueva EPS & $\mathrm{X}$ & & & & & \\
\hline Comparta EAPB & $\mathrm{X}$ & $\mathrm{X}$ & & & & \\
\hline
\end{tabular}

Fuente: Elaboración propia 
ISSN-PRINT

1794-9831

E-ISSN 2322-7028

Vol. 15 No. 2

Jul - Dic 2018

Cúcuta, Colombia
En cuanto al número de EAPB con el servicio de psicología y psiquiatría habilitado, cinco cuentan con estos servicios. Al analizar los servicios y tiempos promedio, se observa que los usuarios asignados a cada profesional de psicología y psiquiatría en promedio al día son 10; quienes acceden al servicio de psicología disponen de un tiempo de atención máximo de 40 minutos, mientras que para los que acceden al servicio de psiquiatría cuentan con 90 minutos por sesión. Por último, se debe precisar que solamente siete de las EAPB tienen habilitado el servicio de acceso a tratamiento farmacológico, con un tiempo máximo de entrega del medicamento de veinte días.

Revisando los convenios con instituciones que tienen centros de atención para internar pacientes con problemas de salud mental de las EAPB, se encontró que la totalidad de las entidades disponen de este servicio habilitado y que cada una posee al menos un convenio, siendo el CRIB la institución que lo presta a la mayoría de las EAPB. Igualmente, las EAPB en su totalidad cuentan con el servicio de seguimiento realizado a pacientes de contrarreferencia y referencia.

Con respecto, a las EAPB que cuentan con el servicio habilitado de atención ambulatoria y rutas para eventos de salud mental, cinco lo ofrecen y disponen de entre cuatro y cinco rutas de atención, utilizándose con más frecuencia las que atienden trastornos alimenticios, consumo de SPA, discapacidad y suicidio.

Respecto a las IPS, que ofrecen la red prestadora de servicios para cada una de las EAPB, se realizó una lista de las que estaban ubicadas en Tunja; también, se identificaron otras redes que prestan sus servicios para Tunja en ciudades como Duitama, Sogamoso y Chiquinquirá, obteniendo un total de 39 instituciones; de las cuales, 32 permitieron acceder a la información, en donde se encontró que ocho ofrecían el servicio de urgencias para salud mental, además cuentan con protocolos que permiten solucionar las dificultades que se puedan presentar. Es importante aclarar que aunque las demás instituciones no prestaban el servicio de urgencias para salud mental, 15 al menos poseían un protocolo específico de salud mental, siendo más frecuentes los procedimientos realizados con consentimiento informado, seguido por el protocolo de atención para víctimas de violencia sexual y el de atención para pacientes con $\mathrm{VIH}+/$ SIDA. Por otra parte, se observó que el servicio de urgencias para salud mental se encuentra en seis instituciones que tienen al menos un profesional en las áreas de psicología, psiquiatría, medicina general, enfermería y auxiliar de enfermería, quienes en su mayoría cuentan con una contratación por prestación de servicios.

Por otra parte, al revisar el servicio de consulta externa para salud mental, se encontró que 22 instituciones cuentan con este servicio, de las cuales 11 tienen contratados Psicólogos generales y 11 Psicólogos especialistas: cinco están especializados en Psicología clínica y 2 en psicología clínica infantil y psicología clínica y de la familia. Respecto al salario de los psicólogos vinculados a las IPS para prestar el servicio de consulta externa, se encontró que la mayoría de los profesionales tienen un contrato por prestación de servicios (OPS) o para cubrir eventos específicos, con una remuneración que oscila entre $\$ 5.000$ a $\$ 13.000$ por evento; así mismo, se comprobó que ocho psicólogos tienen un salario que fluctúa entre $\$ 1^{\prime} 500.000$ y $\$ 2.700 .000$ por mes; sin embargo, se debe aclarar que la mayoría de las IPS no brindaron la información pertinente debido a que no poseen el conocimiento sobre este aspecto. En cuanto a la experiencia profesional, 36 psicólogos de consulta externa tienen entre uno y siete años de experiencia.

Sobre el servicio de consulta externa, nueve de las instituciones tienen el apoyo de profesionales de psiquiatra, medicina general, auxiliar de enfermería, enfermería y terapeuta ocupacional; en este ítem también se encontró que 16 es el número de pacientes atendidos al día por profesionales de psicología; el número de sesiones por paciente para consulta externa de esta misma especialidad está entre 7 y 12, con 30 minutos por sesión.

Sin duda, el servicio de mayor oferta es el de Interconsulta en 27 IPS, seguido por valoración con 21 IPS; por otro lado, tres de las instituciones cuentan con habilitación para acceso a farmacología según la necesidad del usuario, dentro de las cuales se encuentran la Clínica de la Policía y el CRIB en la ciudad de Tunja y en la ciudad de Duitama La Clínica Boyacá, las 29 restantes expresaron que este servicio lo proporciona la EAPB de cada usuario. También, en este ítem se indica que el tiempo promedio de asignación para una cita de consulta externa es de un día y cuatro horas, siendo ocho días lo mínimo que 
debe esperar el usuario para control por psiquiatría y un día para el de psicología.

Con relación a los profesionales de salud mental que apoyan otros procedimientos realizados en las instituciones, se encontró que seis de las IPS cuentan con este apoyo para otros procedimientos, dentro de los que se encuentra con mayor frecuencia la atención domiciliaria al paciente agudo. Respecto, a herramientas o servicios de salud mental, se observó que 23 IPS prestan este servicio y el tiempo máximo que demora el proceso es de 24 horas, después de éste lapso de tiempo es la EAPB la responsable de seguir con la remisión.

En cuanto a los servicios y herramientas para hospitalización en salud mental, se encontró que solo una institución presta este servicio; igualmente, ninguna de las instituciones cuenta con el servicio de hospitalización en unidad especializada para consumidores de sustancias psicoactivas; sin embargo, se descubrió que si no prestan el servicio de hospitalización disponen de algunos servicios como los presentados en la tabla 2 .

Tabla 2. Numero de IPS que brindan servicios y herramientas para hospitalización en salud mental

\begin{tabular}{lc}
\hline \multicolumn{1}{c}{ Servicio } & Numero de IPS \\
\hline Hospitalización en unidad de salud mental & 1 \\
\hline Área exclusiva para el manejo de usuarios con problemas y trastornos en salud mental y consumo SPA & 1 \\
\hline Posee área exclusiva para la hospitalización en salud mental para niños y /o adolescentes & 1 \\
\hline Posee puesto de control de enfermería, con sistema de monitoreo visual y/o vigilancia permanente & 1 \\
\hline Transporte residencial & 0 \\
\hline Terapia electro convulsiva con anestesia relajante & 0 \\
\hline Servicio de Tele psiquiatría & 0 \\
\hline Referencia a servicios de mayor complejidad & 11 \\
\hline Hay recepción de usuarios remitidos desde baja complejidad & 5 \\
\hline Contra remisión de usuarios & 4 \\
\hline Valoración de ingreso y egreso por psiquiatría & 5 \\
\hline Trabajo intersectorial & 4 \\
\hline Internación hospitalaria e internación parcial para la atención al consumidos de SPA & 2 \\
\hline No total de IPS & 11 \\
\hline
\end{tabular}

Fuente: Elaboración propia Fuente

Por su parte el servicio de interconsulta se presta en 27 IPS, las cuales cuentan con profesionales de psicología, psiquiatría y terapeuta ocupacional. Respecto a los convenios con centros de rehabilitación cuatro IPS los tienen: Colombiana de Salud con el CRIB, el Hospital San Rafael en Tunja y en Bogotá la Clínica Santo Tomás. Por otra parte, la clínica de la Policía cuenta con el Hospital San Rafael y el CRIB en Tunja y en Bogotá con la Clínica Inmaculada y el Hospital Central; también, la Universidad Pedagógica y Tecnológica de Colombia tiene convenios con el Hospital San Rafael; y por último, el Hospital Regional de Duitama tiene convenio con el CRIB en Tunja, Fundación Tundama en Duitama y Clínica la paz y Talita Kumi en Bogotá.

\section{Discusión}

Teniendo en cuenta los planteamientos de las resoluciones 2003 del 2014 y 6408 del 2016 (20,21) y considerando que la salud mental no deja de ser un tema aislado, si antes no se realizan investigaciones que promuevan su conocimiento y se propongan estrategias que permitan mejorar las condiciones de los recursos que se tienen sobre la salud mental; es por eso que el presente estudio tiene como objetivo describir el estado actual de los servicios de atención en salud mental brindados por las Instituciones Prestadoras de Servicio (IPS) y los servicios habilitados por las EAPB, con el fin de analizar el 
ISSN-PRINT

1794-9831

E-ISSN 2322-7028

Vol. 15 No. 2

Jul - Dic 2018

Cúcuta, Colombia cumplimiento de lo establecido en las resoluciones propuestas y servir de base para futuros estudios.

En relación a los hallazgos de las EAPB y teniendo en cuenta los tipos de aseguramientos existentes, se encontró que los servicios habilitados en cada entidad se ofrecen para los usuarios con tipo de aseguramiento contributivo y subsidiado; esto se sustenta en el acuerdo 029 de 2011 (22) desarrollado por la Comisión Reguladora de Salud, en el que se expone que se debe dar un aumento en la cobertura de los servicios de salud específicamente para la población con este tipo de aseguramiento.

Es importante resaltar que aunque existan normas que establecen cómo se deben llevar a cabo los servicios de salud mental, el conocimiento de estas es insuficiente, puesto que las entidades competentes no tienen conocimiento de los servicios prestados; al respecto expresaron que la red prestadora de servicios es la encargada de desarrollar este proceso según su juicio. Debido a ese desconocimiento no se logró que la mayoría de las EAPB ofreciera información sobre: el tiempo determinado para consulta, número de sesiones mínimas y máximas por profesional de psicología y de psiquiatría. Teniendo en cuenta este aspecto, se sugiere que tanto las EAPB como las IPS tengan conocimiento de la norma que estipula los tiempos y sesiones promedio que deben brindar a cada uno de los afiliados.

En cuanto, a las ocho EAPB que tienen habilitado el servicio de urgencias para salud mental y que cuentan con al menos una institución que presta este servicio, sin embargo, se debería analizarlas para ver si disponen de los convenios que garanticen un servicio oportuno y de calidad a la totalidad de los usuarios; ya que como lo plantea el Ministerio de Protección Social (23) este servicio es fundamental para las personas, puesto que al presentar una alteración de la integridad física, funcional y/o mental, con cualquier grado de severidad que comprometa su vida o funcionalidad, se requieren acciones oportunas de los servicios de salud, con el fin de conservar la vida y prevenir consecuencias futuras (24).

Si se tiene en cuenta que a principios de 2018 se eliminó el Plan Obligatorio de Salud (POS), que tenía estipulado el manejo de urgencias en salud mental que se debía aplicar a cada usuario, relacionado con los tiempos promedio para internación o remisión y demás servicios, se hace necesario realizar un estudio que permita reconocer los cambios tanto positivos como negativos derivados de la nueva reglamentación.

De la misma manera, se debe tener en cuenta, que si bien las EAPB habilitan los servicios de salud mental, no necesariamente dan cuenta de la calidad de los servicios que prestan, lo cual se puede verificar al momento de analizar la red contratada de servicios, ya que no se identifican estadísticas precisas acerca de los usuarios que se remiten; además, al hacer un promedio de los usuarios que solicitan servicios de salud mental, el número de la red de servicios con las que se tiene convenio es insuficiente; como se puede concluir cumplen con una mínima parte de lo estipulado por la ley 1616 (11) en la que se expone que las EAPB deberán disponer de una red integrada de prestación de servicios de salud mental pública y privada. Por tanto, esta red de servicios generales de salud, debe estar integrada en el marco de las estrategias APS en salud de atención integral, incluyendo la prestación de servicios en todos los niveles de complejidad, con el fin de garantizar una atención oportuna, suficiente, continua, pertinente, de fácil acceso y que cuente con servicios de promoción, prevención, detección temprana, diagnóstico, intervención, tratamiento y rehabilitación en salud mental.

Por otro lado, se debe precisar que solo ocho de las 32 IPS prestan el servicio de urgencias en salud mental, dentro de las que se encuentra cinco privadas. Sin embargo, en el Informe Nacional de Calidad de la APS (25) en el que se hace referencia a la tendencia sobre la prestación de servicio de urgencias principalmente por IPS públicas, difiere en los resultados obtenidos debido a que la mayoría son privadas; al respecto, se podría señalar como una de las explicaciones, es que en este estudio se contó con información de un número mayor de Instituciones.

Aunque no se logró acceder al total de instituciones de la red de servicios de Tunja, la mayoría de estas se encuentran en nivel de atención primario y secundario, y sólo una parte presta los servicios de urgencias para salud mental lo cual representa una gran dificultad, sobre todo cuando la ley 1616 (11) COLPSIC (26), establece que debe ser un servicio básico al que debe tener acceso toda la población, sirviendo como apoyo para identificar y evitar síntomas agudos, antes de que se conviertan en crónicos y generen mayor gasto en hospitalización. 
Respecto a los profesionales, se encontró que quienes prestan este servicio en su mayoría son médicos y enfermeras, percibiéndose como una dificultad, debido a que el servicio de urgencias y demás servicios de salud mental deben desarrollarse bajo el enfoque de APS, con profesionales capacitados en conocimientos y habilidades que les permitan desarrollar, identificar y responder satisfactoriamente a las necesidades de la población $(11,27)$. Cabe mencionar, que si bien los médicos y enfermeras pueden contar con una formación básica en salud mental, sin embargo, no es suficiente al momento de identificar síntomas y generar estrategias de prevención e intervención y por tanto los enfermos se deben derivar a profesionales especializados; razón por la cual es necesaria la actualización permanente con el fin de lograr un primer acercamiento satisfactorio y oportuno ante cualquier situación que se presente en urgencias.

Al respecto, Vignolo et al. (28), proponen que se debería instruir desde la academia a estos profesionales sobre los servicios de salud mental, permitiendo identificar al personal idóneo, mejorando así su desempeño, evitando problemas y costos posteriores a sus pacientes.

De igual manera, se debe resaltar que según los hallazgos del presente estudio, los profesionales en psicología contratados por las IPS para brindar servicios tales como asesoría, valoración, interconsulta y psicoterapia casi en su totalidad no cuentan con la especialidad requerida para el abordaje que implica esta especialidad, ya que es necesario que cuenten con habilidades, destrezas, experiencia y mayor conocimiento, que les permitan responder de manera adecuada y efectiva con el deber como profesionales en el área de la Psicología. De igual forma, deben tener una formación rigurosa en las áreas aplicadas específicamente en Psicología Clínica y de la Salud.

De otra parte, si se tiene en cuenta los planteamientos de la ley 1090, Londoño y Flórez y COLPSIC (18, 29,30 ), es necesario evaluar a los profesionales de psicología, permitiendo identificar sus habilidades, destrezas, y conocimientos; así mismo se deben identificar los factores que limitan o favorecen su labor. Con el objeto de resolver esta situación se puede invitar a instituciones competentes como la Secretaria de Salud y COLPSIC, con el fin de que realicen acciones que permitan dar cumplimiento al decreto, generando evaluaciones y exigencias continuas a los profesionales que trabajan en este ámbito.

En lo que se refiere a la consulta externa solamente 22 de las 32 IPS prestan este servicio, de las cuales 11 cuentan con profesionales especialistas; sin embargo, es importante aclarar que las especialidades que poseen los psicólogos contratados por las IPS son en diversas áreas de la psicología, siendo mínimo el número de especialistas en Psicología Clínica. Teniendo en cuenta lo planteado anteriormente, se debe precisar que si bien las instituciones cumplen con la ley 1090 (18) la cual expone que cualquier psicólogo puede oficiar como clínico sin tener títulos de Especialización o Maestría en Psicología Clínica, se especifica que esto no lo certifica como psicólogo clínico. En concordancia con lo dispuesto en el artículo $2^{\circ}$ la responsabilidad ética sobre la actuación del psicólogo debe fundamentarse en los lineamientos de competencia profesional, teniendo en cuenta los principios deontológicos, entre los cuales está aquel por el cual el psicólogo solamente puede ejercer dentro de sus límites y competencias.

Partiendo de los supuestos anteriores, se considera necesario que los psicólogos que se desempeñan en este campo, reconozcan el compromiso ético y político que tienen como profesionales y la importancia de contribuir al desarrollo de un país más equitativo, concibiendo la salud como un proceso del bien común y un derecho. Es por este motivo, que es esencial el cumplimiento del decreto 1527 en el que se contempla lo importante que es tener una formación rigurosa en las áreas relacionadas específicamente con la salud mental $(29,30)$, por lo cual es necesario motivar a las entidades competentes para que ejecuten las actividades necesarias que incentiven la revisión, mantenimiento, actualización y adquisición de nuevos conocimientos y habilidades, que permitan mejorar y potenciar la práctica profesional en el campo de la Psicología Clínica y de la Salud (31, 32).

Complementando lo anterior, es importante resaltar que las IPS cumplan con la ley Estatutaria 1751 (13) la cual determina que los establecimientos, los servicios y las tecnologías de salud deberán centrarse en las necesidades del usuario y que respondan a los estándares de calidad aceptados por las comunidades científicas; es por este motivo, que se requiere de personal especializado, que se actualice permanentemente mediante educación continua,
E-ISSN 2322-7028

Vol. 15 No. 2

Jul - Dic 2018

Cúcuta, Colombia 
ISSN-PRINT

1794-9831

E-ISSN 2322-7028

Vol. 15 No. 2

Jul - Dic 2018

Cúcuta, Colombia investigación científica y evaluación oportuna de la calidad de los servicios y tecnologías ofrecidos.

En cuanto al número de sesiones brindadas para cada usuario en consulta externa, se encontró un promedio entre 7 y 12 sesiones. Teniendo en cuenta que la mayoría de las IPS tienen el servicio habilitado para psicoterapia, es necesario identificar si con el número de sesiones promedio se logra cumplir con los objetivos terapéuticos que demanda cada usuario, así como determinar cuáles son las razones para continuar o no con el proceso psicoterapéutico; eventos cuyo cumplimiento depende de diversos factores relacionados con el tiempo, el profesional y el costo; razón por la cual se debe tener en cuenta lo que plantean Rondón et al.(33) para quienes es importante tener en cuenta factores como fallas institucionales, dificultades con el terapeuta o el proceso terapéutico y por último, contextos específicos de la persona que impiden el cumplimiento a lo acordado con el profesional.

De acuerdo con estos planteamientos, sería interesante que en futuras investigaciones, se analizaran las razones por las cuales algunos usuarios que acceden al servicio de consulta externa de psicología, den por terminado el tratamiento antes de cumplir con los objetivos del mismo; de esa forma se daría una explicación pertinente que apoye a las instituciones, a fin de proponer e implementar diferentes estrategias que aumenten tanto la continuidad del usuario en su tratamiento, como la eficiencia en el servicio que se le brinda.

Por otra parte, es importante resaltar que 30 minutos son en promedio el tiempo de atención para cada paciente, por tanto, sería importante indagar si este tiempo es suficiente para atenderlo, así como las sesiones a realizarle, de tal forma que generen resultados favorables, para responder de manera adecuada a sus necesidades; esto se encuentra articulado con lo que proponen Painepán y Kühne (34), quienes exponen que el grado de efectividad óptima para los pacientes se encuentra ubicada entre la sesión 8 y 12 de una psicoterapia.

Así mismo se resalta que aunque no se tiene información específica de los usuarios, que permita identificar si hay o no abandono en el proceso psicoterapéutico o ante el servicios ofertados debido a la atención prestada, sería enriquecedor analizar el tipo de contrato de los profesionales; se sabe que la mayoría están contratados por prestación de servicios (OPS), siendo un punto de importancia ya que además de que no cuentan con un horario fijo, el profesional varia constantemente; por otro lado, la mayoría de los profesionales no cuentan con experticia en habilidades clínicas que permitan un mejor desarrollo de sus competencias y por tanto generar resultados favorables en los usuarios.

En un estudio realizado por Salamanca et al.(35) señalan algunos de los factores relacionados con el abandono de la psicoterapia, como son: expectativas de pacientes, formación profesional, características del terapeuta, habilidades clínicas, procesos de derivación, administración, recepción de datos, manejo de la información, cambios constantes del terapeuta, procedimientos y costos de la institución; que si bien no se mencionan en su totalidad en el presente estudio, sería importante investigar más acerca de las consecuencias que trae consigo el abandono prematuro de un proceso de intervención psicológica.

De igual forma, al revisar la remuneración de los profesionales en psicología, se encontró que es de $\$ 5.000$ y $\$ 13.000$ por hora de trabajo o evento presentado, y aunque el Colegio Colombiano de Psicólogos propuso una tabla salarial específica dependiendo de los servicios prestados, contratación, especialización y experiencia; sin embargo, se observa que no se tiene en cuenta, puesto que en la misma se estipula que el salario mínimo para psicólogos generales, con un tiempo de tres meses de experiencia y contratados bajo la modalidad de prestación de servicios debe ser de $\$ 2.068 .362$.

$\mathrm{Al}$ respecto surge la inquietud sobre las estrategias a implementar desde los entes reguladores para que se tenga en cuenta la tabla salarial; por otro lado se debe realizar una indagación respecto a las características sobre la atención de los servicios de salud mental y la forma como los profesionales que prestan este servicio lo realizan y si su calidad responde a la retribución monetaria que reciben.

Complementando lo anterior, el que el profesional en psicología acepte este tipo de salario puede explicarse teniendo en cuenta las tasas de desempleo y la competencia profesional que existe en el medio, ya que según García y Rendón (36) los psicólogos han perdido la estabilidad laboral, debido a la reducción 
de las contrataciones en el sector salud y el aumento en la modalidad de contratación por prestación de servicios o a término no definido; por otro lado, se resalta que son pocos los profesionales que consideran la opción de trabajo independiente y esto hace que aún más se empleen bajo esta modalidad o se encuentren desempleados.

Por su parte, el Ministerio de Salud y Protección Social (24) realizó un análisis del estado de la salud y encontró que solo una ESE cuenta con servicios de psiquiatría en el Departamento de Boyacá, obteniendo una calificación de riesgo "medio", sugiriendo a las autoridades competentes reforzar y complementar los servicios brindados, por ser prestador único de la red pública de salud mental. Al respecto, cabe resaltar que para la presente investigación aún se contaba específicamente con el CRIB para servicios de psiquiátrico y que la habilitación de Hospitalización en el área de salud mental, dispone de siete psiquiatras y un psicólogo con especialización en Clínica y de la familia, lo cual se considera insuficiente de acuerdo a las demandas del servicio y la reglamentación establecida.

Adicionalmente y teniendo en cuenta que el CRIB es la única institución que brinda este servicio y que varias instituciones a nivel de Boyacá cuentan con su apoyo, es importante tener en cuenta los planteamientos de Posada (37), quien reporta que Colombia ocupa el quinto puesto entre los países que presentan mayor prevalencia de las enfermedades de salud mental y que afecta a dos de cada cinco personas; ya que si bien el CRIB es una institución única en su clase a nivel de Boyacá, no cuenta con el sistema de urgencias habilitado y sus servicios son insuficientes ante la demanda actual de la población, generando así una lista larga de usuarios que esperan para acceder a los servicios que se brindan.

Se hace necesario entonces, realizar un estudio que permita describir el estado de los servicios prestados, que dé cuenta sobre la importancia de contratar personal suficiente que permita cubrir las necesidades de Boyacá y que los entes competentes propongan estrategias, como el desarrollo y establecimiento de nuevos centros especializados en el área de salud mental, permitiendo resolver de manera eficaz y oportuna esta problemática presente en el departamento.
Respecto a los resultados encontrados, se puede decir que es importante prestar servicios de salud mental humanizados, ya que como lo plantean Ávila y Yaneth (38), los servicios no cuentan con humanización por que algunas características impiden ofrecerlos de manera oportuna y con calidad como lo son: la poca contratación de profesionales, el tipo de contratación por OPS, el bajo nivel de experticia de los profesionales, el exceso ante la demanda de los servicios y los pocos convenios interinstitucionales, entre otras.

Algo semejante ocurre con las competencias profesionales las cuales deben ser una representación de los diferentes roles y funcionar en una variedad de contextos ocupacionales para diferentes tipos de usuarios; lo que significa que deben estar basadas en los conocimientos, habilidades, destrezas, aptitudes y actitudes aplicadas éticamente; de ahí, que los profesionales que se encuentran vinculados en el área de salud, deban contar con formación específica, que les permita responder oportunamente a las problemáticas que se presentan en esta área; por lo que se propone realizar una investigación que analice sus competencias, habilidades y conocimientos, con el fin de generar estrategias para contratar profesionales idóneos o generar mecanismos que faciliten su crecimiento profesional en pro del mejoramiento de la atención a los usuarios.

Para finalizar, es indispensable que cada EAPB e IPS, cuente con personal que tenga conocimientos suficientes y actualizados sobre los servicios de salud mental que prestan. También se sugiere que se les ofrezca una inducción y orientación sobre esta especialidad. Es por esto, que se propone que en futuras investigaciones la información pueda obtenerse de manera exacta y oportuna; ya que se encontró que los profesionales que respondieron a la información solicitada, pertenecían a varias especialidades y su conocimiento no era completo, lo que implicaba demora en recolección de la información y vacíos en datos que afectan el conocimiento total y la veracidad de los resultados.

\section{Conclusiones}

- Las IPS, ofrecen algunos servicios de salud mental, pero estos no cumplen con el total de las características propuestas en la resolución 2003 de 2014.
E-ISSN 2322-7028

Vol. 15 No. 2

Jul - Dic 2018

Cúcuta, Colombia 
- La mayoría de las IPS evaluadas, no prestan el servicio de urgencias para salud mental.

- Ocho EAPB cuentan con red contratada de servicios para psicología, entre los que se encuentran los de urgencias y atención con internación en convenio con IPS pública.

- Se debe concientizar a los profesionales del área de salud mental sobre la importancia y responsabilidad de tener las competencias necesarias que les permitan brindar servicios oportunos y de calidad a la población, disminuyendo así el predominio de problemas crónicos sobre la salud mental y sus consecuencias.

\section{Conflicto de Intereses}

Las autoras declaran no tener ningún conflicto de intereses.

\section{Referencias Bibliográficas}

1. Ministerio de salud y protección social-Colciencias y Pontificia Universidad Javeriana. Estudio nacional de salud mental Colombia 2015. Bogotá D.C.: Editorial Javegraf.; 2015.

2. Edwards J. Fundamental Facts about Mental Heald. Mental Health Foundation. Reino Unido. [libro en internet]; 2015 [consultado el 27 de Febrero de 2017] Disponible en: https://www.mentalhealth.org. uk/sites/default/files/fundamental-facts-15.pdf

3. WHO's. Investing in mental health: evidence for action. World Health Organization. Ginebr. [libro en internet]; 2013 [consultado el 3 de Agosto de 2017] Disponible en: http://apps.who.int/iris/ bitstream/10665/87232/1/9789241564618 eng.pdf.

4. Santacruz C, Torres N, Gómez C, Matallana D, Borda JP. Construcción de un componente de salud mental para la encuesta nacional. ENSM-2015, Colombia. Rev. Colombiana de Psiquiatría. [Internet]. 2016 [Consultado el 09 de Septiembre de 2017]; 45(S1):19-25. Disponible en http://www.scielo.org. co/pdf/rcp/v45s1/v45s1a04.pdf.

5. Honorato J, Arango C, Sanjuán J, Ros S, Vieta E-Ayuso JL, et al. Presente y futuro de las enfermedades mentales más prevalentes. INESME [libro en internet]; 2009; 45-53. [consultado el 27 de Febrero de 2017]. Disponible en: http://iris.paho.org/xmlui/bitstream/handle/123456789/740/9789275316320. pdf

6. WHO's. Mental health atlas. World Health Organization. Ginebra. Editorial Organización Mundial de la salud; 2011.

7. Organización Mundial de la Salud. Plan de acción sobre salud mental 2013-2020. Ginebra.: Editorial Organización mundial de la salud.; 2013.

8. Alvarez R.¿cuáles son los sistemas de salud más eficientes del mundo?. United Explanations [Internet]. 2013 [Consultado el 20 Febrero de 2017]. Disponible en: http://www.unitedexplanations. org/2013/11/08/los-sistemas-de-salud-mas-eficientes-del-mundo/

9. González AC. Análisis comparativo de los sistemas de salud de Cuba, Canadá y Colombia. [Trabajo de grado Magister]. Bogotá: Universidad Nacional de Colombia; 2014.

10. República de Colombia. Congreso de Colombia. Ley 100 de 1993, diciembre 23, por la cual se crea el sistema de seguridad social integral y se dictan otras disposiciones. Diario Oficial No. 41.148. Bogotá: El Ministerio; 1993.

11. República de Colombia. Congreso de Colombia. Ley 1616 de 2013, por medio de la cual se expide la ley de salud mental y se dictan otras disposiciones. Diario Oficial No. 48.680. Bogotá: El Ministerio; 2013.

12. WHO's. Integrating mental health services into primary he alth care. Wonca. Sigapur.; 2008.

13. República de Colombia. Congreso de Colombia. Ley 1751 de 2015, febrero 16, por medio de la cual 
se regula el derecho fundamental a la salud y se dictan otras disposiciones. Diario Oficial No. 49.427. Bogotá: El Ministerio; 2015.

14. República de Colombia. Congreso de Colombia. Ley 1566 de 2012, julio 31, por la cual se dictan normas para garantizar la atención integral a personas que consumen sustancias psicoactivas y se crea el premio nacional "entidad comprometida con la prevención del consumo, abuso y adicción a sustancias psicoactivas. Diario Oficial No. 48.508. Bogotá: El Ministerio; 2012.

15. República de Colombia. Superintendencia del subsidio familiar. Ordenanza 0023 de 2010, Septiembre 11, aadoptar el Modelo de "Atención Primaria en Salud con Enfoque Familiar APS Salud Familiar. Departamento de Boyacá; 2011.

16. Henao S, Restrepo V, Alzate A, González C. Percepción sobre el acceso a los servicios de salud mental que tienen los residentes de tres municipios de Antioquia: Revista Facultad Nacional de Salud Pública; 2009; 27(3); 272-280.

17. Berbesi Y, Bareño J. Diagnóstico de oferta de servicios en salud mental en municipios de Antioquia. Scielo, Hacia la Promoción de la Salud; 2010; 15(1):41-54.

18. República de Colombia. Congreso de Colombia. Ley 1090 de 2006, Septiembre 06, por la cual se reglamenta el ejercicio profesional de Psicología, se dicta el Código Deontológico y Bioético y otras disposiciones. Diario Oficial No. 46.383. Bogotá: El Ministerio; 2006.

19. República de Colombia. Ministerio de Salud y Protección Social. Resolución 8430 de 1993, Octubre 4, por la cual se establecen las normas científicas, técnicas y administrativas para la investigación en salud. Colombia; 1993.

20. República de Colombia. Ministerio de Salud y Protección Social. Resolución 2003 de 2014, Mayo 28, por la cual se definen los procedimientos y condiciones de inscripción de los prestadores de servicios de salud y de habilitación de servicios de salud. Colombia; 2014.

21. República de Colombia. Ministerio de Salud y Protección Social. Resolución 6408 de 2016, Diciembre 26, por la cual se modifica el Plan de Beneficios en Salud con cargo a la unidad de pago por capitación (UPC). Colombia; 2016.

22. República de Colombia. Ministerio de Salud y Protección Social. Acuerdo 029 de 2011, Diciembre 28, por el cual se sustituye el Acuerdo 028 de 2011 que define, aclara y actualiza integralmente el Plan Obligatorio de Salud. Colombia; 2011.

23. Ministerio de la Protección Social. Guía de Atención en Salud Mental en Emergencias y Desastres: Colombia. Bogotá D.C.: Editorial Impresol; 2011.

24. Ministerio de Salud y Protección Social. Indicadores básicos de salud en Boyacá. [Libro en Internet]. Boyacá: ASIS; 2011 [consultado el 30 de Marzo de 2017]. Disponible en: https://www.minsalud.gov. co/plandecenal/mapa/Analisis-de-Situacion-Salud-Boyaca-2011.pdf

25. Ministerio de Protección Social. Informe Nacional de Calidad de Atención en Salud 2015. [Artículo de Internet]. 2015 [consultado el 09 de Septiembre de 2017]. Disponible en: https://www.minsalud.gov. co/sites/rid/Lists/BibliotecaDigital/RIDE/DE/DIJ/informe-nal-calidad-atencion-salud-2015.pdf

26. Colegio Colombiano de Psicólogos. Salud mental y atención primaria en salud: una necesidad apremiante para el caso Colombiano. [Artículo en Internet]. Médicos sin fronteras: Colombia; 2016 [consultado el 30 de Junio de 2017]. Disponible en: http://www.colpsic.org.co/aym image/files/ Articulo_MSF y ColPsic 14-10-2015 DC 7 final.pdf

27. República de Colombia. Ministerio de Salud y Protección Social. Resolución 429 de 2016, Febrero 17, por medio de la cual se adopta la política de Atención Integral en Salud. Diario oficial No. 49.794. Bogotá. El Ministerio de Salud y Protección Social.

28. Vingolo J, Vacarezza M, Álvarez C y Sosa A. Niveles de atención, de prevención y de atención primaria de la salud. Rev. Scielo [Internet]. 2011 [Consultado el 05 de Mayo de 2017]; 33(1):11-14. Disponible en: http://www.scielo.edu.uy/pdf/ami/v33n1/v33n1a03.pdf 
ISSN-PRINT

$1794-9831$

E-ISSN 2322-7028

Vol. 15 No. 2

Jul - Dic 2018

Cúcuta, Colombia

29. Londoño C, Florez L. Formación en psicología de la Salud en Colombia; Universidad Católica de Colombia. Psychologia: avances de la disciplina [Internet]. 2008 [Consultado el 07 de Mayo de 2017]; 4(1):55-61. Disponible en: revistas.usb.edu.co/index.php/Psychologia/article/download/1158/950

30. República de Colombia. Congreso de Colombia. Ley 1527 de 2002, Julio 24, por el cual se establecen los estandares de calidad en programas profesionales de oregrado en Psicologia. Diario oficial No. 44.883. Bogotá: El Ministerio; 2002.

31. Colegio Colombiano de Psicólogos. Perfil y competencias del psicólogo en Colombia, en el contexto de la salud. [Artículo en Internet]. Bogotá; 2014. [Consultado el 24 de Julio de 2017] Disponible en: https:// www.minsalud.gov.co/sites/rid/Lists/BibliotecaDigital/RIDE/VS/TH/Psicologia_Octubre2014.pdf

32. Colegio Oficial de Psicólogos. Perfiles profesionales del psicólogo. Psicología Clínica y de la Salud [Internet]. 2014 [Consultado el 24 de Julio de 2017]; 22-56. Disponible en: https:/www.ucm.es/data/ cont/docs/315-2014-02-07-Perfil\%20psicologia\%20clinica\%20y\%20de\%201a\%20salud.pdf

33. Rondón AP, Otálora IL, Salamanca Y. Factores que influyen en la deserción terapéutica de los consultantes de un centro universitario de atención psicológica. Rev. International Journal of Psychological Research [Internet]. 2009 [consultado el 20 de Octubre de 2017]; 2(2):137-147. Disponible en: http://revistas.usb.edu.co/index.php/IJPR/article/viewFile/869/620

34. Painepán B, Kügne W. Efectividad según duración de la psicoterapia en un centro de atención psicológica para universitarios. Rev. SUMMA PSICOLOGICA [Internet]. 2012 [consultado el 27 de Octubre de 2017]; 9(1):47-52. Disponible en: http://pepsic.bvsalud.org/pdf/summa/v9n1/a05.pdf

35. Salamanca Y, Otalora I, Rondon A, Martinez Y. Factores asociados al abandono psicoterapéutico de los consultantesdedosinstitucionesColombianas. Rev.dePsicologíadeArequipa[Internet].2016[consultado el 20 de Octubre de 2017]; 6(1):305-318. Disponible en: https://www.researchgate.net/profile/Yenny Salamanca_Camargo2/publication/314282658 Factores asociados_al abandono psicoterapeutico de_los_consultantes_de_dos_instituciones_colombianas/links/58bf6c57a6fdccff $7 \mathrm{~b} 1 \mathrm{fa} 0005 /$ Factoresasociados-al-abandono-psicoterapeutico-de-los-consultantes-de-dos-instituciones-colombianas.pdf

36. García B, Rendón B. Condiciones laborales del psicólogo en Medellín (Colombia) Fundación Universitaria Los Libertadores Bogotá. [Internet]. 2013 [consultado el 28 de Octubre de 2017]; 7:144159. Disponible en: http://www.ulibertadores.edu.co/images/documentos-institucionales/documentos/ cifras-2013.pdf?x68241

37. Posada JA. La salud mental en Colombia. Rev. Scielo [Internet]. 2013[consultado el 05 de Marzo de 2017]; 33(4). Disponible en: http://www.scielo.org.co/pdf/bio/v33n4/v33n4a01.pdf

38. Ávila E, Yaneth D. Humanización de los servicios de salud mental en un establecimiento de sanidad militar. [Tésis en Internet]. Bogotá: Universidad Santo Tomas; 2017 [consultado el 28 de Octubre de 2017]. Disponible en: http://repository.usta.edu.co/bitstream/handle/11634/3975/Avilaedwin2017. pdf? sequence $=1$ 